\title{
Validación de un tamiz precoz de preeclampsia en una población mexicana
}

\author{
Héctor Oviedo-Cruz ${ }^{*}$, Erika R. Carrasco-Blancas ${ }^{1}$ y Marco A. Cortes-Martínez²
}

${ }^{1}$ Departamento de Medicina Materno-Fetal, Centro Médico para Atención Fetal Especializada, Hospital Español de México; ${ }^{2}$ Departamento de Bioquímica y Calidad, Laboratorio CEMAFE S.A. de C.V. Ciudad de México, México

\section{Resumen}

Antecedentes: Ninguna prueba de tamiz de preeclampsia ha sido validada en nuestro país. Objetivo: Evaluar el ajuste y rendimiento del algoritmo bayesiano FMF 4.0 en una población mexicana. Método: Estudio de cohorte en embarazos de feto único, según el modelo de riesgos en competencia para preeclampsia. El riesgo a priori se calculó por historia clínica. La presión arterial media (PAM), el índice de pulsatilidad medio de la arteria uterina (IPmAUt) y la proteína plasmática A asociada al embarazo (PAPP-A) se midieron a las 11-14 semanas de gestación con metodología estandarizada. El valor de cada marcador se transformó en múltiplos de la mediana (MoM) por el algoritmo original y el corregido a la población. Se aplicaron la distribución normal multivariante y el teorema de Bayes para las probabilidades posprueba. Resultados: Hubo un 5.0\% (40/807) de preeclampsia. Las distribuciones sin preeclampsia fueron PAM 0.927 vs. 0.991 ${ }_{b}$ UtAmPI $0.895_{a}$ vs. $1.030_{b}$ y PAPP-A $0.815_{a}$ vs. $0.963_{b}(p<0.001)$. El área bajo la curva fue $0.821_{a}$ vs. $0.823_{b}$. Para una detección del $69 \%$, los puntos de corte fueron 1 en $240_{a}$ y 1 en $120_{b}$, a falsos positivos del $31 \%_{a}$ y $29 \%_{b}$. Conclusiones: El modelo debe ajustarse a la población diana.

PALABRAS CLAVE: Preeclampsia. Pruebas de tamiz en suero materno. Ecografía Doppler. Presión arterial. Control prenatal. México.

\section{Validation of a preeclampsia early screening test in a Mexican population}

\section{Abstract}

Background: No preeclampsia screening test has been validated in our country. Objective: To assess the fit and performance of the FMF 4.0 Bayesian algorithm in a Mexican population. Method: Cohort study in singleton pregnancies, according to the competing risks model for preeclampsia. A priori risk was calculated based on medical history. Mean blood pressure (MBP), uterine artery mean pulsatility index (UtAmPI) and pregnancy-associated plasma protein A (PAPP-A) were measured at 11-14 weeks of gestation using standardized methodology. The value of each marker was transformed into multiples of the median (MoM) by the original ${ }_{a}$ algorithm and by the population correction ${ }_{b}$. Multivariate normal distribution and Bayes theorem were applied to obtain the post-test probabilities. Results: There was 5.0\% (40/807) preeclampsia. Distributions in women without preeclampsia were $\mathrm{MBP}=0.927_{a}$ vs. $0.991_{b}, \mathrm{UtAmPI}=0.895_{a}$ vs. $1.030_{b}$, and PAPP-A $=0.815_{a}$ vs $0.963_{b}(p<0.001)$. The AUC was $0.821_{a}$ and $0.823_{b}$. For a detection rate of $69 \%$, cutoff points were 1 in $240_{a}$ and 1 in $120_{b}$, with false positive rates of $31 \%$ and $29 \%{ }_{b}$. Conclusions: The model has to be adjusted to the target population.

KEY WORDS: Preeclampsia. Maternal serum screening tests. Doppler ultrasonography. Blood pressure. Prenatal care. Mexico.

Correspondencia:

*Héctor Oviedo-Cruz

E-mail: HectorOC@ @emafe.org.mx

0016-3813/๑ 2021 Academia Nacional de Medicina de México, A.C. Publicado por Permanyer. Este es un artículo open access bajo la licencia CC BY-NC-ND (http://creativecommons.org/licenses/by-nc-nd/4.0/).
Fecha de recepción: 31-01-2021

Fecha de aceptación: 13-04-2021 


\section{Introducción}

La preeclampsia afecta al $2-8 \%$ de los embarazos en el mundo y es causa principal de morbilidad y mortalidad tanto materna como perinatal' ${ }^{1}$. En México es causa de muerte materna a pesar del control prenatal regular ${ }^{2}$. Las dosis bajas de aspirina iniciadas a las 16 semanas de gestación o antes son profilaxis eficaz en embarazos de alto riesgo ${ }^{3}$. Debido a que la cobertura por historia clínica es baja ${ }^{4}$, se desarrollaron pruebas de marcadores múltiples ${ }^{5-7}$ con mejor desempeño que las guías clínicas ${ }^{8}$. La efectividad de prescribir aspirina profiláctica con base en un tamiz precoz de preeclampsia ha sido recientemente probada $^{9}$, incluso adoptada por la Federación Internacional de Ginecología y Obstetricia (FIGO) ${ }^{10}$.

El rendimiento de estas pruebas ha resultado subóptimo en poblaciones diferentes de las que originaron el modelo ${ }^{11}$, excepto cuando los marcadores fueron corregidos para la población diana ${ }^{12}$.

Uno de estos algoritmos, ampliamente distribuido por su gratuidad, es el de la Fundación de Medicina Fetal de Londres, Reino Unido (FMF) ${ }^{5,6,13}$. Su versión más reciente, FMF $4.0(2018)^{14}$, fue liberada en marzo 2019 y se cuentan al menos 277 usuarios registrados en México (https://fetalmedicine.org/lists/map/certified/Ut); pero no se incluyó población mexicana en su creación ni se ha documentado validación alguna en ella.

El objetivo de este estudio es evaluar el rendimiento del algoritmo FMF $4.0^{14}$ en una población mexicana con y sin corrección de marcadores.

\section{Materiales y métodos}

Se realizó un estudio en una cohorte de estudios perinatales ${ }^{15}$. El reclutamiento fue de enero 2012 a diciembre 2019 y se obtuvo la aprobación de los comités científico y bioético de la sede, apegándose a la Declaración de Helsinki de la Asociación Médica Mundial para las investigaciones médicas en seres humanos.

Los criterios de inclusión fueron embarazo de feto único con vitalidad y longitud corona-cauda (LCC) entre 45 y $84 \mathrm{~mm}\left(11^{+1}\right.$ a $14^{+1}$ semanas de gestación), con datos completos para el estudio y que se haya registrado el nacimiento de recién nacido fenotípicamente normal, vivo o muerto, con al menos 24 semanas de gestación o más. Se excluyeron embarazos con defectos cromosómicos, no cromosómicos y los que tuvieron aborto espontáneo o inducido antes de las 24 semanas.

\section{Historia clínica}

Se obtuvo por cuestionario autoadministrado, verificado por interrogatorio y codificado operativamente según la $\mathrm{FMF}^{16}$.

El peso y estatura maternos se obtuvieron con báscula y estadímetro clínicos convencionales analógicos calibrados.

La edad de la gestación se estableció por LCC por ecografía según el método de Robinson corregido por Loughna, et al..$^{17}$.

\section{Medición de la presión arterial media}

Estandarizada por la $\mathrm{FMF}^{18}$ y adoptada por la $\mathrm{FIGO}^{10}$, congruente con las recomendaciones internacionales actuales ${ }^{19}$, requirió el uso del brazalete mediano único proporcionado con cada dispositivo; los de otros calibres no están disponibles en el mercado local y se ha asumido corrección del sesgo braquial por peso materno ${ }^{20}$.

Los dispositivos electrónicos automáticos fueron marca Omron ${ }^{\circledast}$ (Japón), modelo HEM-7121, que cumple con el requisito $510(\mathrm{k})$ de la Food and Drug Administration (FDA). Se calibraron periódicamente. El personal encargado de las mediciones fue entrenado y supervisado constantemente.

\section{Medición del índice de pulsatilidad medio de las arterias uterinas}

Se emplearon ecógrafos marca Voluson (GE Healthcare Technologies ${ }^{\circledR}$ ) modelos E10, E6, S8, 730 Expert, Logiq P5 y Logiq 3 Expert; todos con transductores transabdominales y transvaginales multifrecuencia, bajo mantenimiento preventivo constante. Los exámenes fueron realizados por dos operadores certificados y auditados periódicamente (fetalmedicine.org) siguiendo metodología estandarizada ${ }^{21}$.

\section{Medición de proteína plasmática $A$ asociada al embarazo}

Se emplearon analizadores automatizados, calibrados y controlados, basados en inmunoensayos ultrasensibles y aprobados por la FMF, a saber: BRAHMS Kryptor compact PLUS (ThermoFisher ${ }^{\circledR}$ ) por emisión 
amplificada de criptato resuelta en tiempo, Cobas e411 $\left(\right.$ Roche $\left.^{\circledR}\right)$ por electroquimioluminiscencia, y DELFIAXpress (PerkinElmer ${ }^{\circledR}$ ) emisión amplificada de europio resuelta en tiempo.

Las muestras fueron suero de sangre venosa periférica (braquial), procurando la estabilidad del analito $^{22}$ y la validez temporal ${ }^{23}$.

\section{Desenlaces de interés}

El resultado de cada embarazo fue obtenido del acta de nacimiento, defunción, de alumbramiento, del expediente clínico o por anamnesis de la paciente al regresar al centro por nuevo embarazo. Los registros de mujeres con hipertensión asociada al embarazo fueron revisados para clasificar preeclampsia o no preeclampsia de acuerdo con la Sociedad Internacional para el Estudio de la Hipertensión en el Embarazo (ISSHP) ${ }^{24}$.

\section{Análisis estadístico}

Se aplicó el algoritmo FMF $4.0^{14}$, basado en el modelo de riesgos en competencia ${ }^{25}$ para preeclampsia. El riesgo a priori se estableció por historia clínica ${ }^{16}$. Los valores de cada marcador se estandarizaron en múltiplos de la mediana (MoM) tanto por el algoritmo original ${ }^{14}\left(\mathrm{MoM}_{a}\right)$ como por las medianas específicas de la población $\left(\mathrm{MoM}_{\mathrm{b}}\right)$; estas están en prensa (10.6084/m9.figshare.12268328, $\quad 10.6084 / \mathrm{m} 9$. figshare.12991322), excepto PAPP-A ${ }^{23,26}$. Se centró la configuración de MoM esperados ${ }^{13} \mathrm{a}<37$ semanas y se calcularon las funciones de densidad de probabilidad normal multivariante de cada marcador y sus combinaciones (escenarios) con base en los $\log _{10}(\mathrm{MoM})$ de cada modelo y las matrices de covarianza para cada desenlace. Los cocientes de probabilidad por escenario se obtuvieron de las densidades de probabilidad correspondientes y se aplicaron al riesgo a priori para obtener la probabilidad bayesiana posprueba.

La distribución MoM sin preeclampsia se auditó según los criterios de la FMF (https://fetalmedicine. org/research/audit/). Las líneas de regresión de MoM por semanas de gestación al parto por preeclampsia se compararon con el modelo hipotético ${ }^{13,14}$ mediante la t de Student para coeficientes ${ }^{27}$.

Se evaluó el rendimiento posprueba por escenarios de cada modelo mediante las curvas $\mathrm{ROC}^{28}$.

A manera de piloto se calculó y graficó el desempeño de las guías del Colegio Americano de
Ginecólogos y Obstetras (American College of Obstetricians and Gynecologists, ACOG) ${ }^{29}$ y del Instituto Nacional del Reino Unido para la Excelencia en Salud y Cuidado (National Institute for Health and Care Excellence, NICE) ${ }^{30}$.

Se exploró la representatividad de las probabilidades posprueba de cada modelo por inspección de sus curvas de calibración ${ }^{31}$.

Se probaron los supuestos de normalidad, linealidad y homocedasticidad para decidir aplicar estadísticos paramétricos, no paramétricos o transformar las variables para análisis.

El nivel de significancia fue $\alpha=0.05$ para las pruebas estadísticas (grado de significación [p]).

El tamaño de la muestra se basó tanto en el poder estadístico ${ }^{32}$ para el área mínima reportada bajo la curva ROC: $0.733^{14}$ (596 observaciones), como en una frecuencia del $5.4 \%$ para preeclampsia en la Ciudad de México $^{33}$ y una sensibilidad del $65.6 \%$ para la prueba en población distinta de la que la originó12; $^{2}$ se calculós2 $n=741$ con 40 incidencias de preeclampsia.

La base de datos fue creada desde los programas Astraia $^{\circledR}$ (Alemania) y Excel $^{\circledR}$ (EE.UU.), disponible mediante petición razonable al autor de correspondencia. El análisis estadístico se realizó en los programas Excel $^{\circledR}$ (EE.UU.) y MATLAB ${ }^{\circledR}$ (EE.UU.) con licencias vigentes, los códigos de programación son propiedad del laboratorio.

\section{Resultados}

La incidencia de preeclampsia fue 40/807 (5.0\%; intervalo de confianza del 95\% [IC 95\%]: 3.6-6.3). Fueron 29/40 (73\%) nacimientos < 37 semanas por preeclampsia y $118 / 767(15 \%)$ sin preeclampsia $(p<0.001$, prueba exacta de Fisher). Las características de la muestra se presentan en la tabla 1.

La distribución de los marcadores sin preeclampsia se presenta en la figura 1. Los valores centrales del modelo original ${ }_{a}$ se ubicaron fuera de \pm 0.2 desviaciones estándar (DE) del hipotético:

$\mathrm{PAM}=0.927 \mathrm{MoM}_{a}=-0.9 \mathrm{DE}$

$\mathrm{IPm}=0.895 \mathrm{MoM}_{a}=-0.4 \mathrm{DE}$

PAPP-A $=0.815 \mathrm{MoM}_{a}=0.4 \mathrm{DE}$

Los valores centrales del modelo corregido ${ }_{b}$ para la población local estuvieron dentro del óptimo $\pm 0.1 \mathrm{DE}$ del hipotético:

PAM $=0.991 \mathrm{MoM}_{b}=-0.1 \mathrm{DE}$

$\mathrm{IPm}=1.030 \mathrm{MoM}_{b}=+0.1 \mathrm{DE}$

PAPP-A $=0.963 \mathrm{MoM}_{b}=-0.1 \mathrm{DE}$. 
Tabla 1. Características maternas y de la gestación en los grupos de estudio

\begin{tabular}{|c|c|c|c|}
\hline Variables & No preeclampsia & Preeclampsia & valor $p$ \\
\hline Edad materna (años) ${ }^{\dagger}$ & $33.0(30.0 ; 35.9)$ & $34.4(31.0 ; 39.0)$ & $0.032^{*}$ \\
\hline Peso materno $(\mathrm{kg})^{\dagger}$ & $61.4(55.4 ; 68.6)$ & $63.6(58.8 ; 74.2)$ & 0.060 \\
\hline Talla materna $(\mathrm{cm})^{\dagger}$ & $160.0(156.0 ; 164.0)$ & $161.5(159.0 ; 164.6)$ & 0.101 \\
\hline Índice de masa corporal ${ }^{\dagger}$ & $23.9(21.8 ; 26.8)$ & $24.0(23.2 ; 39.0)$ & 0.134 \\
\hline Edad de gestación (semanas) ${ }^{\dagger}$ & $12.7(12.3 ; 13.3)$ & $12.7(12.1 ; 13.2)$ & 0.568 \\
\hline $\begin{array}{l}\text { Etnia }^{\ddagger} \\
\text { Caucásico/hispano } \\
\text { Afrocaribeño } \\
\text { Surasiático } \\
\text { Este asiático } \\
\text { Otro, mixto }\end{array}$ & $\begin{array}{l}674(87.9) \\
3(0.4) \\
2(0.3) \\
16(2.1) \\
72(9.4)\end{array}$ & $\begin{array}{l}34(85.0) \\
0(0.0) \\
0(0.0) \\
0(0.0) \\
6(15.0)\end{array}$ & $\begin{array}{l}0.619 \\
1.000 \\
1.000 \\
1.000 \\
0.265\end{array}$ \\
\hline $\begin{array}{l}\text { Concepción }{ }^{\ddagger} \\
\text { Espontánea } \\
\text { Fertilización in vitro } \\
\text { Inductores de la ovulación }\end{array}$ & $\begin{array}{l}686(89.4) \\
46(6.0) \\
35(4.6)\end{array}$ & $\begin{array}{l}31(77.5) \\
9(22.5) \\
0(0.0)\end{array}$ & $\begin{array}{l}0.034^{*} \\
0.001^{*} \\
0.410\end{array}$ \\
\hline $\begin{array}{l}\text { Hábito de fumar } \\
\text { Fumadora } \\
\text { Dejó de fumar } \\
\text { No fuma }\end{array}$ & $\begin{array}{c}16(2.1) \\
83(10.8) \\
668(87.1)\end{array}$ & $\begin{array}{c}1(2.5) \\
4(10.0) \\
35(87.5)\end{array}$ & $\begin{array}{l}0.582 \\
1.000 \\
1.000\end{array}$ \\
\hline $\begin{array}{l}\text { Paridad }{ }^{\ddagger} \\
\text { Nulípara } \\
\text { Paridad sin PE } \\
\text { Paridad con PE previa }\end{array}$ & $\begin{array}{c}562(73.3) \\
195(25.4) \\
10(1.3)\end{array}$ & $\begin{array}{l}32(80.0) \\
1(2.5) \\
7(17.5)\end{array}$ & $\begin{aligned} & 0.462 \\
< & 0.001^{*} \\
< & 0.001^{*}\end{aligned}$ \\
\hline $\begin{array}{l}\text { Historia médica }{ }^{\ddagger} \S \\
\text { Hipertensión crónica } \\
\text { Lupus eritematoso sistémico } \\
\text { Síndrome antifosfolípidos } \\
\text { Historia familiar de } \mathrm{PE}^{\ddagger}\end{array}$ & $\begin{array}{l}6(0.8) \\
1(0.1) \\
6(0.8) \\
9(1.2)\end{array}$ & $\begin{array}{l}2(5.0) \\
0(0.0) \\
0(0.0) \\
4(10.0)\end{array}$ & $\begin{array}{l}0.056 \\
1.000 \\
1.000 \\
0.003^{*}\end{array}$ \\
\hline
\end{tabular}

Las comparaciones entre los grupos de estudio se realizaron mediante la prueba chi cuadrada o exacta de Fisher para variables categóricas y la $\mathrm{U}$ de Mann-Whitney para variables continuas.

*Estadísticamente significativo.

${ }^{\dagger}$ Mediana (IQ).

$\neq n(\%)$.

\$No hubo embarazadas con diabetes mellitus en ninguno de los grupos de estudio.

PE: preeclampsia.
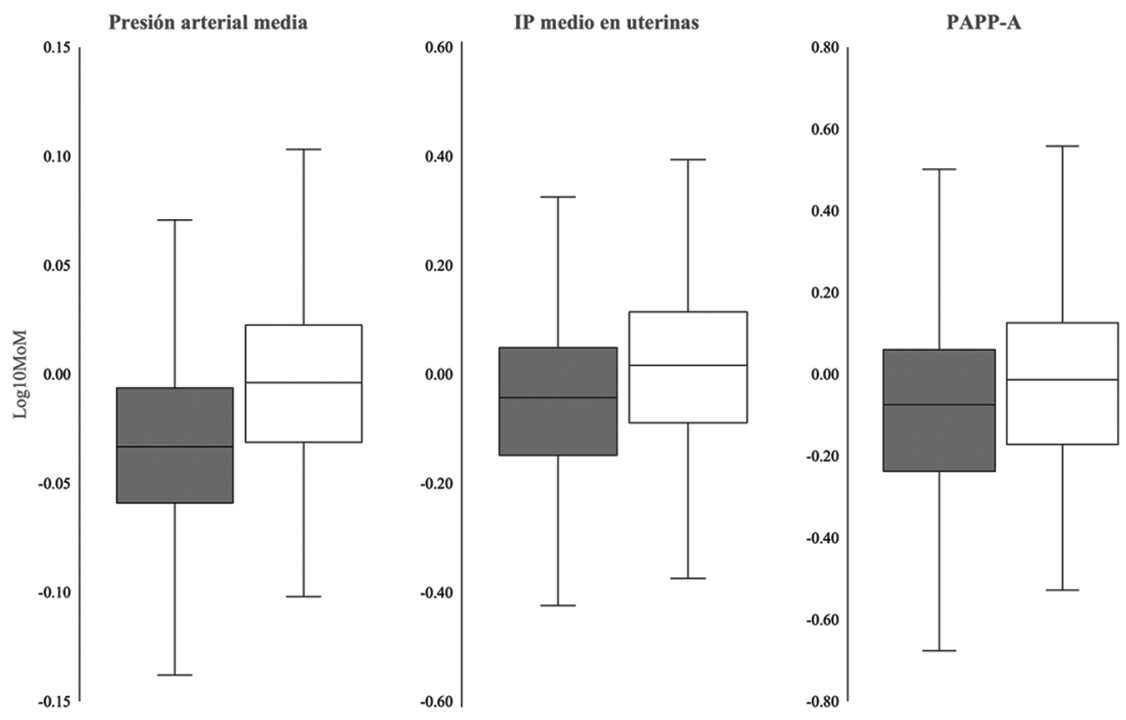

Figura 1. Distribución de los marcadores transformados en logaritmo base 10 de los múltiplos de la mediana (MoM) dentro del grupo sin preeclampsia ( $n=767)$; en gráficos de caja y bigote, a la par el modelo original ${ }^{14}$ y el corregido para la población local (caja blanca); todos los pares fueron significativamente diferentes $(p<0.001$, prueba $t)$.

IP: índice de pulsatilidad; PAPP-A: proteína plasmática A del embarazo. 


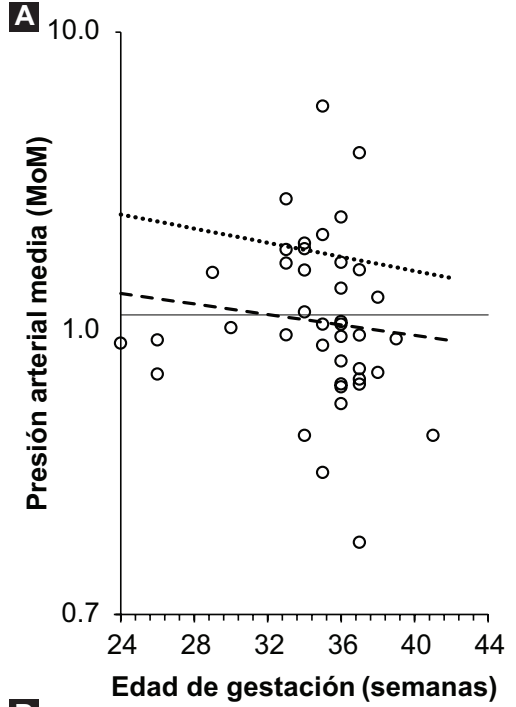

D

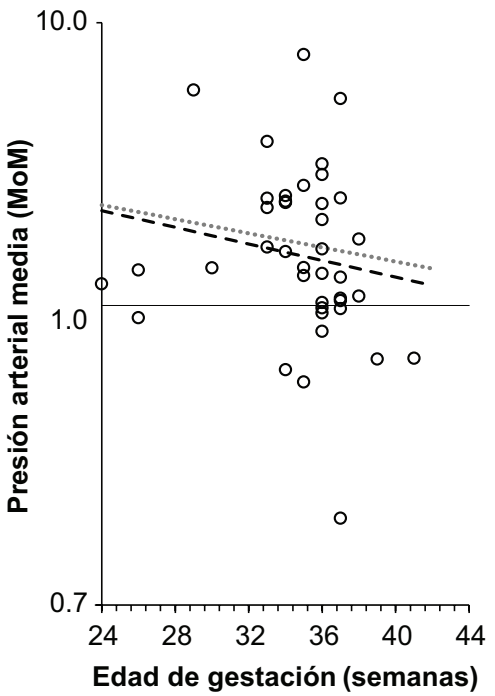

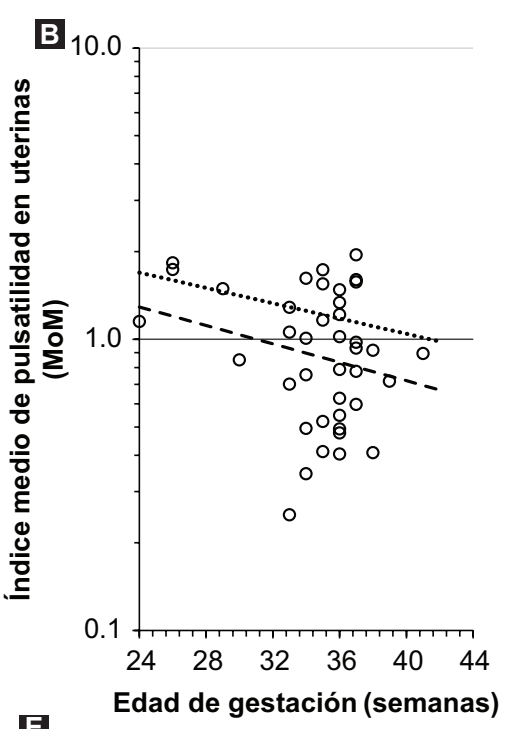

$\mathbf{E}$
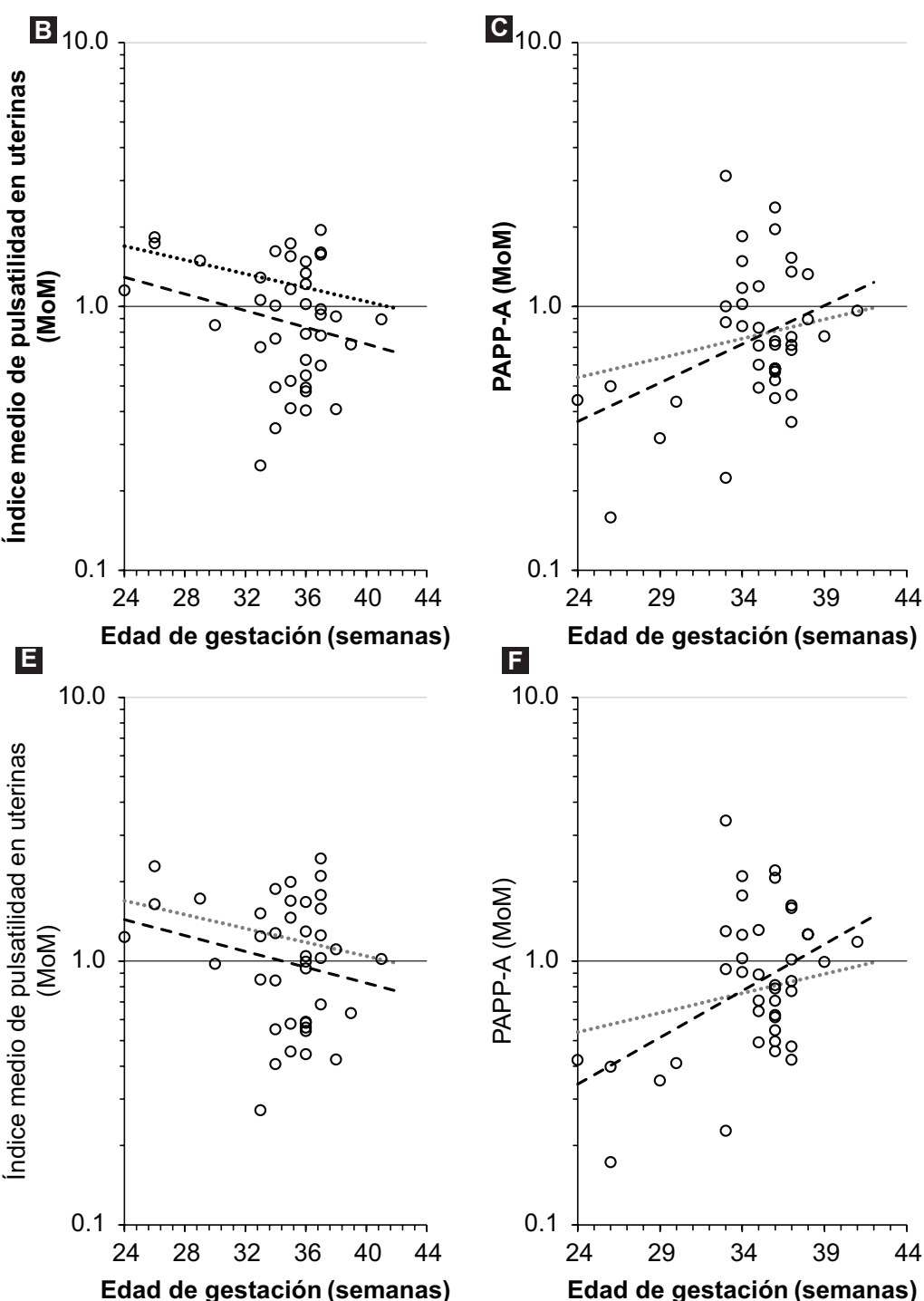

Figura 2. Distribución de los múltiplos de la mediana (MoM) por edad de gestación al nacimiento por preeclampsia (o). Línea de regresión propia de la muestra (- -) vs. línea hipotética ${ }^{13}$ ('). La línea horizontal (-) se refiere a 1.0 MoM. El modelo original de MoM en superior (A, B y C); el modelo corregido para la población en inferior (D, E y F).

PAPP-A: proteína plasmática A del embarazo

La distribución de los MoM por edad de gestación al parto por preeclampsia se presenta en la figura 2 con las líneas de regresión hipotéticas y las propias de la muestra tanto para el modelo original ${ }_{a}$ como para el corregido $o_{b}$. La línea de la PAM resultó paralela $(p=0.226)$ pero significativamente menor a la hipotética en el modelo original ${ }_{a}(p=0.001)$; en contraste, la línea del modelo corregido ${ }_{b}$ no resultó significativamente diferente de la hipotética (pendiente $p=0.406$ e intersección $p=0.744$ ). La línea del IP medio de arterias uterinas en el modelo original ${ }_{a}$ mostró una pendiente más pronunciada, estadísticamente marginal ( $p=0.062)$; mas no su intersección $(p=0.318)$. En el modelo corregido del IP medio de arterias uterinas no se encontró diferencia significativa (pendiente $p=0.188$ e intersección $p=0.617$ ). Las líneas de PAPP-A en ambos modelos resultaron significativamente diferentes en pendiente e intersección $(p<0.001)$.

Las curvas ROC por marcador y sus combinaciones se ilustran en la figura 3 tanto para preeclampsia < 37 semanas como para preeclampsia global; cada modelo por separado con el piloto comparativo de los criterios $\mathrm{ACOG}^{29}$ y NICE${ }^{30}$. Se tabularon puntos de corte relevantes de las curvas ROC en la tabla 2. 

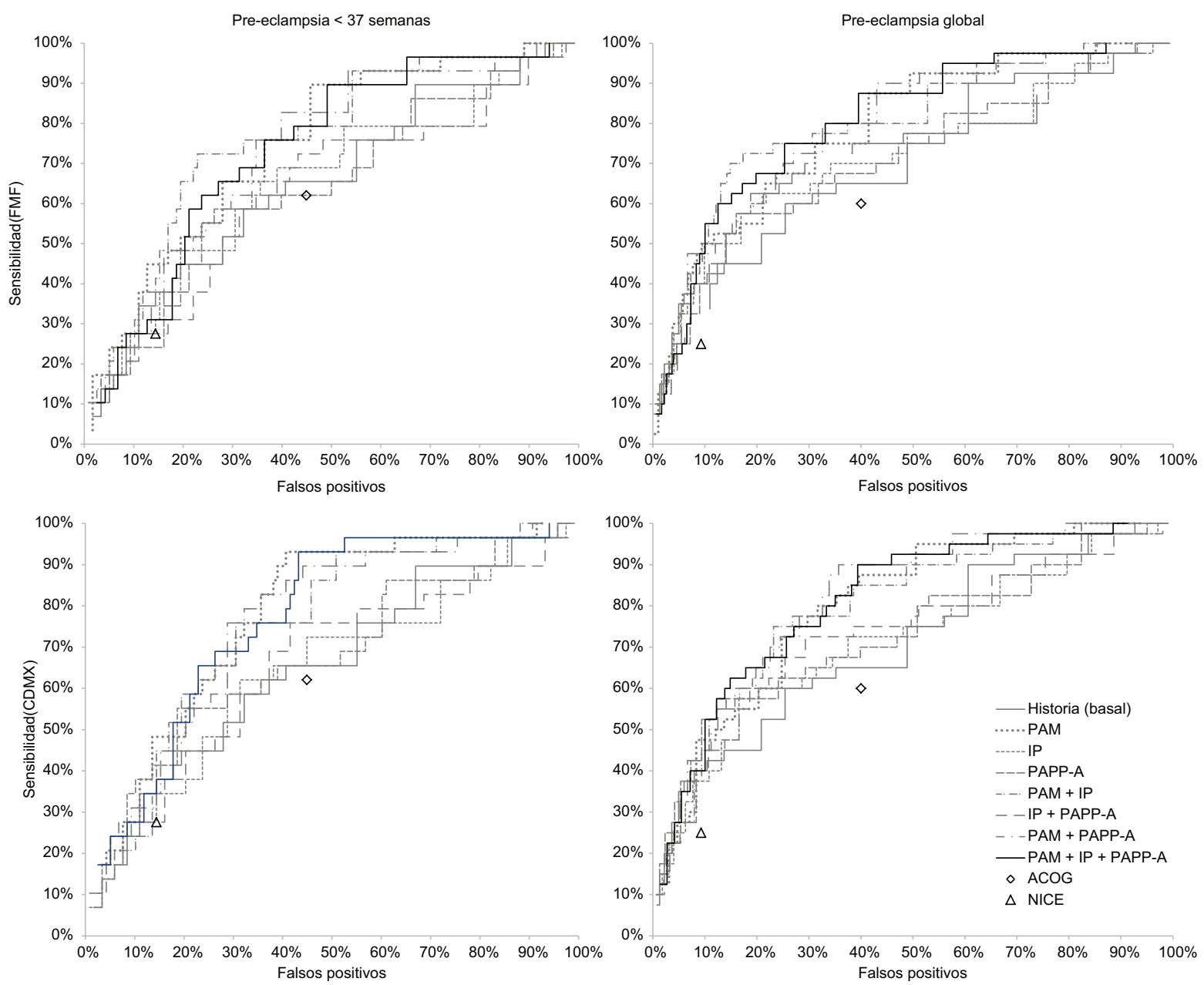

Figura 3. Curvas ROC para preeclampsia < 37 semanas (izquierda) y preeclampsia global (derecha), modelo original ${ }^{14}$ y el corregido para la población local mexicana (inferior).

PAM: presión arterial media; IP: índice de pulsatilidad medio en arterias uterinas; PAPP-A: proteína plasmática A del embarazo; ACOG: Colegio Americano de Ginecólogos y Obstetras (American College of Obstetricians and Gynecologists) ${ }^{29}$; NICE: Instituto Nacional del Reino Unido para la Excelencia en Salud y Cuidado (National Institute for Health and Care Excellence) ${ }^{30}$.

Las líneas de calibración de la prueba combinada total, cada modelo por separado, se representan en la figura 4.

\section{Discusión}

No había información hasta ahora del desempeño del algoritmo FMF $4.0^{14}$ en nuestro país. El análisis por marcador y sus combinaciones es particularmente valioso, porque en nuestro medio varía su disponibilidad.

Otra aportación clave de nuestro estudio fue probar que el modelo original ${ }_{a}$ de medianas FMF-2018 ${ }^{14}$ del algoritmo en estudio no es válido para nuestra población. Los autores desaconsejamos el uso de esta calculadora en México sin corrección poblacional, a menos que sea temporal (v. infra). La corrección de marcadores bioquímicos es posible en los paquetes de cómputo para tamiz de aneuploidías del primer trimestre ${ }^{26}$, mas no para la PAM ni el IP medio de arterias uterinas. Hemos optado por generar nuestra aplicación con las medianas corregidas (v. supra); su validez externa estará limitada por la estandarización de procesos y las diferencias regionales del país.

El modelo corregido de la PAM fue el que mejor reproducibilidad presentó con respecto de lo esperado $^{13}$ y lo reportado por otros ${ }^{12}$. Por esta prueba de validez y la simplicidad del método, debe ampliarse esta línea de investigación.

El modelo corregido ${ }_{b}$ de PAPP-A tuvo una pendiente más pronunciada de lo esperado hacia preeclampsia muy temprana ${ }^{13}$; siendo la corrección con 
Tabla 2. Desempeño de los marcadores y sus combinaciones para preeclampsia < 37 semanas y preeclampsia global, según el modelo original ${ }^{14}$ y el corregido para la población local

\begin{tabular}{|c|c|c|c|c|c|c|c|}
\hline Preeclampsia < 37 semanas & & Detección & Falsos + & & & & \\
\hline ACOG & & $62(46-78)$ & $45(28-61)$ & & & & \\
\hline NICE & & $28(13-42)$ & $14(3-26)$ & & & & \\
\hline \multirow[t]{3}{*}{ Método de tamiz } & \multirow[t]{3}{*}{ Área bajo la curva } & \multicolumn{6}{|c|}{ Punto de corte } \\
\hline & & \multicolumn{2}{|c|}{1 en 60} & \multicolumn{2}{|c|}{1 en 120} & \multicolumn{2}{|c|}{1 en 240} \\
\hline & & Detección & Falsos + & Detección & Falsos + & Detección & Falsos + \\
\hline Historia (a priorı) & $0.671(0.570-0.772)$ & $34(19-50)$ & $11(6-16)$ & $52(35-68)$ & $28(21-35)$ & $90(80-100)$ & $74(67-81)$ \\
\hline \multicolumn{8}{|c|}{ Modelo original } \\
\hline $\begin{array}{l}\text { Historia + } \\
+ \text { + Presión arterial media } \\
+ \text { IP medio en arterias uterinas } \\
\text { + PAPP-A } \\
\text { + Presión arterial media + IP medio } \\
\quad \text { en arterias uterinas }\end{array}$ & $\begin{array}{l}0.765(0.680-0.850) \\
0.672(0.571-0.773) \\
0.659(0.556-0.762) \\
0.793(0.714-0.872)\end{array}$ & $\begin{array}{l}28(13-42) \\
28(13-42) \\
38(22-54) \\
24(10-38)\end{array}$ & $\begin{array}{c}8(3-12) \\
9(5-14) \\
19(13-26) \\
6(2-10)\end{array}$ & $\begin{array}{l}48(32-65) \\
59(42-75) \\
62(46-78) \\
38(22-54)\end{array}$ & $\begin{array}{c}17(11-23) \\
31(23-38) \\
40(32-48) \\
13(7-18)\end{array}$ & $\begin{array}{l}66(50-81) \\
72(58-87) \\
86(75-98) \\
72(58-87)\end{array}$ & $\begin{array}{l}30(22-37) \\
52(44-60) \\
71(64-79) \\
27(20-34)\end{array}$ \\
\hline $\begin{array}{l}+ \text { IP medio en arterias uterinas + } \\
\text { PAPP-A }\end{array}$ & $0.651(0.547-0.755)$ & $31(16-46)$ & $17(11-23)$ & $62(46-78)$ & $35(27-43)$ & $76(62-90)$ & $58(50-67)$ \\
\hline $\begin{array}{l}\text { + Presión arterial media + PAPP-A } \\
\text { + Presión arterial media + IP medio } \\
\text { en arterias uterinas + PAPP-A }\end{array}$ & $\begin{array}{l}0.746(0.658-0.834) \\
0.755(0.668-0.842)\end{array}$ & $\begin{array}{l}34(19-50) \\
28(13-42)\end{array}$ & $\begin{array}{l}14(8-19) \\
8(4-13)\end{array}$ & $\begin{array}{l}52(35-68) \\
52(35-68)\end{array}$ & $\begin{array}{l}25(18-32) \\
20(14-27)\end{array}$ & $\begin{array}{l}76(62-90) \\
69(54-84)\end{array}$ & $\begin{array}{l}35(27-43) \\
31(24-39)\end{array}$ \\
\hline
\end{tabular}

\section{Modelo corregido a la población}

Historia +

+ Presión arterial media

+ IP medio en arterias uterinas

+ PAPP-A

$0.765(0.680-0.850) \quad 45(28-61)$

$15(9-21) \quad 59(42-75) \quad 25(18-33) \quad 90(80-100) \quad 47(39-56)$

$0.660(0.557-0.763) \quad 34(19-50) \quad 14(9-20) \quad 62(46-78) \quad 38(30-46) \quad 76(62-90) \quad 60(52-68)$

+ Presión arterial media + IP medio

$38(22-54)$

$10(5-15) \quad 59(42-75) \quad 33(25-41)$

$86(75-98) \quad 68(60-75)$

en arterias uterinas

+ IP medio en arterias uterinas + PAPP-A

+ Presión arterial media + PAPP-A $\quad 0.777(0.695-0.859) \quad 48(32-65) \quad 18(12-24) \quad 66(50-81) \quad 26(19-33) \quad 93(85-100) \quad 49(41-57)$

+ Presión arterial media + IP medio $0.785(0.705-0.865) \quad 52(35-68) \quad 18(12-24) \quad 69(54-84) \quad 29(21-36) \quad 93(85-100) \quad 43(35-51)$ en arterias uterinas + PAPP-A

\begin{tabular}{|c|c|c|c|c|c|c|c|}
\hline Preeclampsia global & & Detección & Falsos + & & & & \\
\hline ACOG & & $60(44-76)$ & $40(24-56)$ & & & & \\
\hline NICE & & $25(11-39)$ & $9(0-19)$ & & & & \\
\hline \multirow[t]{3}{*}{ Método de tamiz } & \multirow[t]{3}{*}{ Área bajo la curva } & \multicolumn{6}{|c|}{ Punto de corte } \\
\hline & & \multicolumn{2}{|c|}{1 en 60} & \multicolumn{2}{|c|}{1 en 120} & \multicolumn{2}{|c|}{1 en 240} \\
\hline & & Detección & Falsos + & Detección & Falsos + & Detección & Falsos + \\
\hline Historia (a priori) & $0.726(0.659-0.793)$ & $35(22-48)$ & $5(4-6)$ & $53(38-67)$ & $21(18-23)$ & $93(85-100)$ & $69(67-72)$ \\
\hline
\end{tabular}

Historia +

+ Presión arterial media

+ IP medio en arterias uterinas

+ PAPP-A

+ Presión arterial media + IP medio en arterias uterinas

+ IP medio en arterias uterinas + PAPP-A

+ Presión arterial media + PAPP-A

+ Presión arterial media + IP medio en arterias uterinas + PAPP-A

\begin{tabular}{|c|c|c|c|c|c|c|}
\hline $0.801(0.747-0.855)$ & $30(17-43)$ & $4(3-5)$ & $50(36-64)$ & $9(8-11)$ & $65(52-78)$ & $23(20-26)$ \\
\hline $0.737(0.671-0.803)$ & $33(19-46)$ & $5(4-7)$ & $58(44-71)$ & $17(15-19)$ & $73(60-85)$ & $46(43-49)$ \\
\hline $0.732(0.666-0.798)$ & $40(26-54)$ & $9(7-11)$ & $60(46-74)$ & $27(24-30)$ & 85 (75-95) & $64(61-67)$ \\
\hline $0.887(0.852-0.922)$ & $20(9-31)$ & $3(2-4)$ & $43(29-56)$ & $8(6-10)$ & $70(57-83)$ & $17(15-19)$ \\
\hline $.742(0.677-0.807)$ & $33(19-46)$ & $8(6-10)$ & $63(49-76)$ & $22(19-24)$ & 78 (66-89) & $50(46-53)$ \\
\hline 10.14 & $35(22-48)$ & $5(4-7)$ & $50(36-64)$ & $13(11-15)$ & 85) & $27(2$ \\
\hline $821(0.771-0$ & $23(11-34)$ & $4(3-5)$ & $55(41-69)$ & $10(8-12)$ & $68(54-81)$ & $20(18-$ \\
\hline
\end{tabular}


Tabla 2. Desempeño de los marcadores y sus combinaciones para preeclampsia < 37 semanas y preeclampsia global, según el modelo original ${ }^{14}$ y el corregido para la población local (continuación)

\begin{tabular}{|c|c|c|c|c|c|c|c|}
\hline \multicolumn{8}{|c|}{ Modelo corregido a la población } \\
\hline Historia + & & & & & & & \\
\hline $\begin{array}{l}\text { + Presión arterial media } \\
\text { + IP medio en arterias uterinas }\end{array}$ & $\begin{array}{l}0.808(0.756-0.860) \\
0.728(0.661-0.795)\end{array}$ & $\begin{array}{l}48(33-62) \\
38(24-51)\end{array}$ & $\begin{array}{l}8(7-10) \\
8(6-9)\end{array}$ & $\begin{array}{l}60(46-74) \\
60(46-74)\end{array}$ & $\begin{array}{l}20(18-23) \\
26(23-29)\end{array}$ & $\begin{array}{l}88(78-97) \\
80(69-91)\end{array}$ & $\begin{array}{l}43(40-46) \\
57(54-61)\end{array}$ \\
\hline+ PAPP-A & $0.747(0.683-0.811)$ & $38(24-51)$ & $5(4-7)$ & $58(44-71)$ & $20(18-23)$ & $83(72-93)$ & $61(58-64)$ \\
\hline $\begin{array}{l}\text { + Presión arterial media + IP medio } \\
\text { en arterias uterinas }\end{array}$ & $0.812(0.760-0.864)$ & $45(31-59)$ & $9(8-11)$ & $68(54-81)$ & $21(18-24)$ & $85(75-95)$ & $40(37-43)$ \\
\hline $\begin{array}{l}\text { + IP medio en arterias uterinas + } \\
\text { PAPP-A }\end{array}$ & $0.747(0.683-0.811)$ & $40(26-54)$ & $8(7-10)$ & $68(54-81)$ & $25(23-28)$ & $80(69-91)$ & $54(51-57)$ \\
\hline + Presión arterial media + PAPP-A & $0.825(0.776-0.874)$ & $45(31-59)$ & $9(7-11)$ & $65(52-78)$ & $20(17-22)$ & $90(82-98)$ & $42(38-45)$ \\
\hline $\begin{array}{l}\text { + Presión arterial media + IP medio } \\
\text { en arterias uterinas + PAPP-A }\end{array}$ & $0.823(0.752-0.894)$ & $53(38-67)$ & $10(8-12)$ & $65(52-78)$ & $20(17-22)$ & $90(82-98)$ & $39(36-43)$ \\
\hline
\end{tabular}

ACOG: Colegio Americano de Ginecólogos y Obstetras (American College of Obstetricians and Gynecologists); NICE; Instituto Nacional del Reino Unido para la Excelencia en Salud y Cuidado (National Institute for Health and Care Excellence); IP: índice de pulsatilidad; PAPP-A: proteína plasmática A del embarazo.
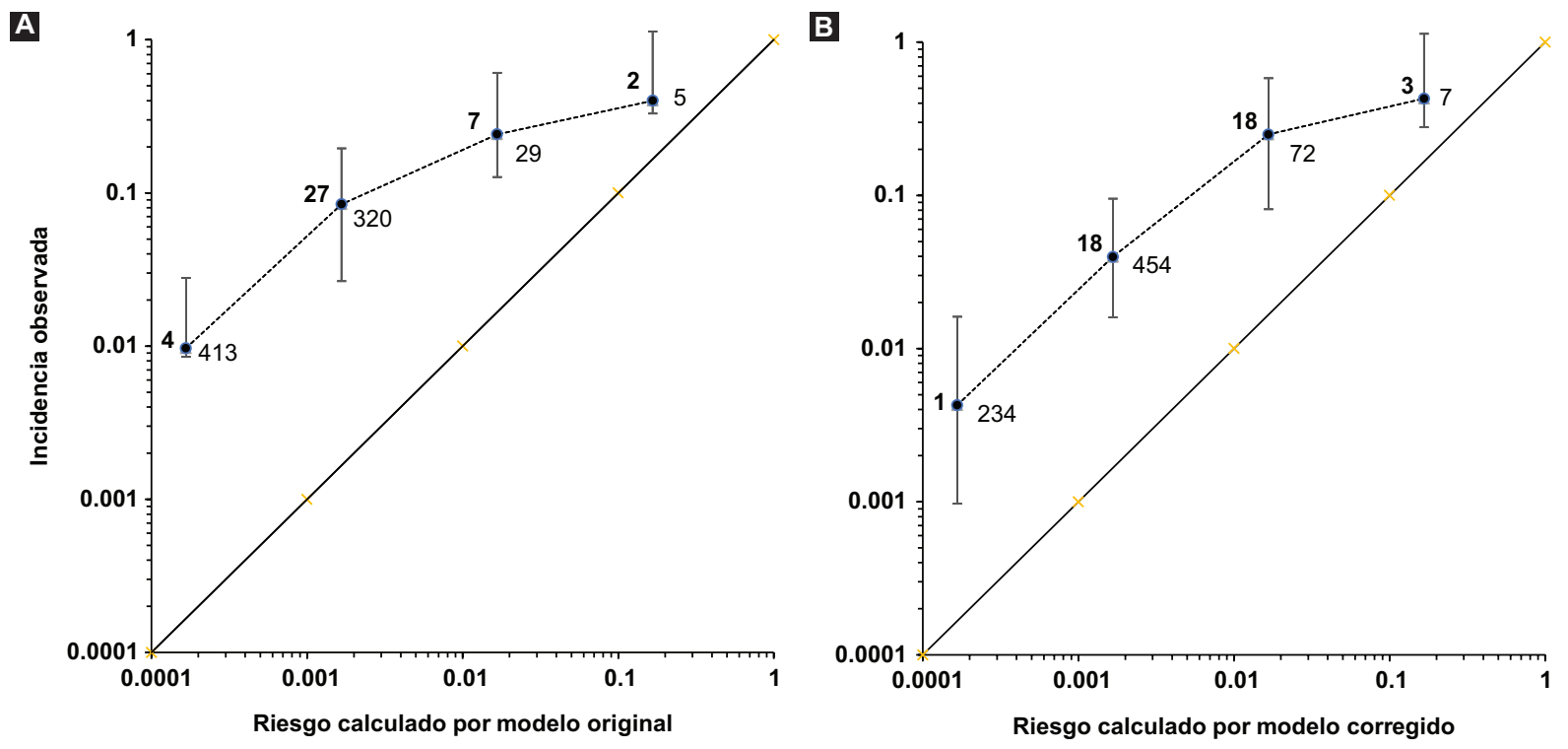

Figura 4. Curvas de calibración para el tamiz combinado de preeclampsia < 37 semanas. Líneas de calibración (- -) para el tamiz de preeclampsia por historia + presión arterial media + índice de pulsatilidad medio en arterias uterinas + proteína plasmática A del embarazo: modelo origina ${ }^{14}$ (A) y modelo corregido (B) para los múltiplos de la mediana (MoM) de la población mexicana local (derecha). La diagonal indica el escenario idóneo.

metodología diferente de la PAM, se justifica investigar el desarrollo del modelo local.

La versión FMF $4.0^{14}$ no tiene punto de corte predeterminado, dejando esta decisión al usuario final. Una aportación útil de nuestro estudio fue la característica ROC, que permite seleccionar el punto de corte por escenario y según las metas en centros similares al nuestro. De la tabla ROC se puede proponer incluso, como solución temporal a la falta de corrección poblacional, un punto de corte mayor para cada escenario.
El piloto comparativo con las guías $\mathrm{ACOG}^{29}$ y NICE ${ }^{30}$ frente al modelo bayesiano multivariante resultó similar a lo reportado por otros, con desempeño menor que el algoritmo $\mathrm{FMF}^{11}$, pero se requiere estudio específico.

Las características de la muestra, las tasas de falsos positivos y la curva de calibración son consistentes con sesgo muestral para embarazos de alto riesgo. El diseño del estudio se basó en el modelo de riesgos en competencia ${ }^{25,34}$; por tanto, los nacimientos sin preeclampsia no fueron necesariamente normales, 
tuvieron diversas indicaciones médicas u obstétricas, con hasta un $15 \%$ de prematuridad en esta muestra de la cohorte mayor. Esto explica mayores tasas de falsos positivos para tasas de detección esperadas de la prueba ${ }^{12-14}$; entonces, también debe considerarse la composición poblacional para seleccionar los puntos de corte prácticos.

Un logro esencial de nuestro estudio fue el código de programación creado ex profeso, resultado del dominio teórico y práctico del algoritmo. Con esto y el seguimiento de la cohorte será posible generar el modelo local de riesgos en competencia para preeclampsia y de otros desenlaces perinatales adversos.

\section{Conclusiones}

Se desaconseja el uso de la calculadora FMF 4.0 14 en México sin corrección de marcadores a la población diana. Se recomienda codificar una aplicación con las medianas locales y mejor aún desarrollar el modelo propio de riesgos en competencia para preeclampsia.

\section{Agradecimientos}

Los autores agradecen al equipo laboral del Centro Médico para Atención Fetal Especializada y Laboratorio CEMAFE S.A. de C.V.

\section{Financiamiento}

La presente investigación no ha recibido ninguna beca específica de agencias de los sectores públicos, comercial o sin ánimo de lucro.

\section{Conflicto de intereses}

Los autores declaran no tener conflicto de intereses.

\section{Responsabilidades éticas}

Protección de personas y animales. Los autores declaran que para esta investigación no se han realizado experimentos en seres humanos ni en animales.

Confidencialidad de los datos. Los autores declaran que han seguido los protocolos de su centro de trabajo sobre la publicación de datos de pacientes.
Derecho a la privacidad y consentimiento informado. Los autores declaran que en este artículo no aparecen datos de pacientes.

\section{Bibliografía}

1. Duley L. The global impact of pre-eclampsia and eclampsia. Semin Perinatol. 2009;33:130-7.

2. Evaluación Estratégica sobre Mortalidad Materna en México 2010: características sociodemográficas que obstaculizan a las mujeres embarazadas su acceso efectivo a instituciones de salud. México, Ciudad de México: CONEVAL; 2012.

3. Bujold E, Roberge S, Lacasse Y, Bureau M, Audibert F, Marcoux S, et al. Prevention of preeclampsia and intrauterine growth restriction with aspirin started in early pregnancy: a meta-analysis. Obstet Gynecol. 2010;116:402-14.

4. Al-Rubaie ZTA, Askie LM, Hudson HM, Ray JG, Jenkins G, Lord SJ. Assessment of NICE and USPSTF guidelines for identifying women at high risk of pre-eclampsia for tailoring aspirin prophylaxis in pregnancy: An individual participant data meta-analysis. Eur J Obstet Gynecol Reprod Biol. 2018;229:159-66.

5. Poon LC, Kametas NA, Maiz N, Akolekar R, Nicolaides KH. First-trimester prediction of hypertensive disorders in pregnancy. Hypertension. 2009;53:812-8.

6. Akolekar R, Syngelaki A, Poon L, Wright D, Nicolaides KH. Competing risks model in early screening for preeclampsia by biophysical and biochemical markers. Fetal Diagn Ther. 2013;33:8-15.

7. Crovetto $F$, Figueras $F$, Triunfo $S$, Crispi $F$, Rodriguez-Sureda V Peguero A, et al. Added value of angiogenic factors for the prediction of early and late preeclampsia in the first trimester of pregnancy. Fetal Diagn Ther. 2014;35:258-66.

8. O'Gorman N, Wright D, Poon LC, Rolnik DL, Syngelaki A, de Alvarado M, et al. Multicenter screening for pre-eclampsia by maternal factors and biomarkers at 11-13 weeks' gestation: comparison with NICE guidelines and ACOG recommendations. Ultrasound Obstet Gynecol. 2017;49:756-60.

9. Rolnik DL, Wright D, Poon LC, O'Gorman N, Syngelaki A, de Paco Matallana C, et al. Aspirin versus placebo in pregnancies at high risk for preterm preeclampsia. N Engl J Med. 2017;377:613-22.

10. Poon LC, Shennan A, Hyett JA, Kapur A, Hadar E, Divakar H, et al. The International Federation of Gynecology and Obstetrics (FIGO) initiative on pre-eclampsia: A pragmatic guide for first-trimester screening and prevention. Int J Gynecol Obstet. 2019;145:1-33.

11. Oliveira N, Magder LS, Blitzer MG, Baschat AA. First-trimester prediction of pre-eclampsia: external validity of algorithms in a prospectively enrolled cohort. Ultrasound Obstet Gynecol. 2014;44:279-85.

12. Chaemsaithong P, Pooh RK, Zheng M, Ma R, Chaiyasit N, Tokunaka M, et al. Prospective evaluation of screening performance of first-trimester prediction models for preterm preeclampsia in an Asian population. Am J Obst Gynecol. 2019;221:650.e1-e16.

13. O'Gorman N, Wright D, Syngelaki A, Akolekar R, Wright A, Poon LC, et al. Competing risks model in screening for preeclampsia by maternal factors and biomarkers at 11-13 weeks gestation. Am J Obst Gynecol. 2016;214:103.e1-e12.

14. Tan MY, Syngelaki A, Poon LC, Rolnik DL, O'Gorman N, Delgado JL, et al. Screening for pre-eclampsia by maternal factors and biomarkers at 11-13 weeks' gestation. Ultrasound Obstet Gynecol. 2018;52:186-95.

15. Oviedo-Cruz H, Hernandez-Paredez J, Ruiz-Ramirez AV. Tamiz prenatal de aneuploidías en el primer trimestre: auditoria a un centro de medicina fetal con laboratorio especializado en Mexico. Ginecol Obstet Mex. 2015;83:259-76.

16. Wright D, Syngelaki A, Akolekar R, Poon LC, Nicolaides KH. Competing risks model in screening for preeclampsia by maternal characteristics and medical history. Am J Obstet Gynecol. 2015;213:62 e1-e10.

17. Loughna P, Chitty L, Evans T, Chudleigh T. Fetal size and dating: Charts recommended for clinical obstetric practice. Ultrasound. 2009;17:160-6.

18. Poon LCY, Zymeri NA, Zamprakou A, Syngelaki A, Nicolaides KH. Protocol for measurement of mean arterial pressure at 11-13 weeks' gestation. Fetal Diagn Ther. 2012;31:42-8.

19. Unger T, Borghi C, Charchar F, Khan NA, Poulter NR, Prabhakaran D, et al. 2020 International Society of Hypertension Global Hypertension Practice Guidelines. Hypertension. 2020;75:1334-57.

20. $\mathrm{Ng} \mathrm{CM}$, Badon SE, Dhivyalosini M, Hamid JJM, Rohana AJ, Teoh AN, et al. Associations of pre-pregnancy body mass index, middle-upper arm circumference, and gestational weight gain. Sex Reprod Healthc. 2019;20:60-5.

21. Gomez O, Figueras F, Fernandez S, Bennasar M, Martinez JM, Puerto $B$, et al. Reference ranges for uterine artery mean pulsatility index at 11-41 weeks of gestation. Ultrasound Obstet Gynecol. 2008;32:128-32. 
22. Cruz J, Cruz G, Minekawa R, Maiz N, Nicolaides KH. Effect of temperature on free beta-human chorionic gonadotropin and pregnancy-associated plasma protein-A concentration. Ultrasound Obstet Gynecol. 2010;36:141-6.

23. Wright D, Spencer K, Kagan KK, Torring N, Petersen OB, Christou A, et al. First-trimester combined screening for trisomy 21 at 7-14 weeks' gestation. Ultrasound Obstet Gynecol. 2010;36:404-11.

24. Brown MA, Magee LA, Kenny LC, Karumanchi SA, McCarthy FP, Saito $S$, et al. The hypertensive disorders of pregnancy: ISSHP classification, diagnosis \& management recommendations for international practice. Pregnancy Hypertens. 2018;13:291-310.

25. Kalbfleisch JD, Prentice RL. Competing risks and multistate models. En: Kalbfleisch JD, Prentice RL, editores. The statistical analysis of failure time data. $2^{\text {nd }}$ Ed. Hoboken, New Jersey, EE.UU.: John Wiley \& Sons; 2002. pp. 247-77.

26. Oviedo-Cruz H, Reyes-Mendoza M, Mestizo-Reyes V. Corrección de medianas de la fracción beta libre de gonadotrofina coriónica humana y proteína plasmática A del embarazo del primer trimestre para una muestra de población mexicana. Ginecol Obstet Mex. 2017; 85:787-98.
27. Andrade JM, Estevez-Perez MG. Statistical comparison of the slopes of two regression lines: A tutorial. Anal Chim Acta. 2014;838:1-12.

28. Steyerberg EW, Vickers AJ, Cook NR, Gerds T, Gonen M, Obuchowski N, et al. Assessing the performance of prediction models: a framework for traditional and novel measures. Epidemiology. 2010;21:128-38.

29. ACOG Practice Bulletin No. 202: Gestational hypertension and preeclampsia. Obstet Gynecol. 2019;133:e1-e25.

30. National Guideline Alliance (UK). Hypertension in pregnancy: diagnosis and management. London: National Institute for Health and Care Excellence (UK); junio 2019.

31. Wright D, Tan MY, O'Gorman N, Poon LC, Syngelaki A, Wright A, et al. Predictive performance of the competing risk model in screening for preeclampsia. Am J Obstet Gynecol. 2019;220:199. e1-e13.

32. Hajian-Tilaki K. Sample size estimation in diagnostic test studies of biomedical informatics. J Biomed Inform. 2014;48:193-204.

33. Ceron M, Harlow, Sanchez C, Nunez RM. Risk factors for pre-eclampsia/eclampsia among working women in Mexico City. Paediatr Perinat Epidemiol. 2001;15:40-6.

34. Wright D, Akolekar R, Syngelaki A, Poon LC, Nicolaides KH. A competing risks model in early screening for preeclampsia. Fetal Diagn Ther. 2012;32:171-8. 